\title{
Glivenko sequent classes in the light of structural proof theory
}

\author{
Negri, Sara
}

2016

Negri , S 2016, ' Glivenko sequent classes in the light of structural proof theory ', Archive for Mathematical Logic , vol. 55 , no. 3 , pp. 461-473 . https://doi.org/10.1007/s00153-016-0474-y

http://hdl.handle.net/10138/178041

https://doi.org/10.1007/s00153-016-0474-y

gnu_lgpl

acceptedVersion

Downloaded from Helda, University of Helsinki institutional repository.

This is an electronic reprint of the original article.

This reprint may differ from the original in pagination and typographic detail.

Please cite the original version. 


\title{
Glivenko sequent classes in the light of structural proof theory
}

\author{
Sara Negri \\ Department of Philosophy \\ University of Helsinki \\ sara.negri@helsinki.fi
}

\begin{abstract}
In 1968, Orevkov presented proofs of conservativity of classical over intuitionistic and minimal predicate logic with equality for seven classes of sequents, what are known as Glivenko classes. The proofs of these results, important in the literature on the constructive content of classical theories, have remained somehow cryptic. In this paper, direct proofs for more general extensions are given for each class by exploiting the structural properties of G3 sequent calculi; for five of the seven classes the results are strengthened to height-preserving statements, and it is further shown that the constructive and minimal proofs are identical in structure to the classical proof from which they are obtained.
\end{abstract}

Keywords: Sequent calculus, Glivenko classes, conservativity of classical over intuitionistic theories, predicate logic with equality and functions, proof analysis.

Mathematical Subject Classification: 03F05, 18C10, $18 \mathrm{~B} 15$.

\section{Introduction}

Glivenko's well known result of 1929 established that a negated propositional formula provable in classical logic is provable intuitionistically. Similar later transfers from classical to intuitionistic provability therefore fall under the nomenclature of Glivenko-style results: these are results about classes of formulas for which classical provability gives intuitionistic provability. The interest in isolating such classes lies in the fact that it may be easier to prove theorems by the use of classical rather than intuitionistic logic. Further, since a proof in intuitionistic logic can be associated to a lambda term and thus obtain a computational meaning, such results have more recently been grouped together 
under the wide conceptual umbrella concerned with the "computational content of classical theories".

The first systematic study of these classes appeared in a paper by Orevkov of 1968. Orevkov's results are not about formulas but about sequents with at most one formula in the succedent. The classes are defined in terms of absence of positive or negative occurrences of logical constants in the sequent and establish that for seven precisely defined classes of sequents, classical derivability in the predicate calculus with equality is equivalent to intuitionistic derivability.

These old results, although central for the investigations on the computational contents of classical theories, have not received the attention they deserve, in part because of their rather complex proofs. There have been, however, isolated efforts in the literature to obtain the results first proved by Orevkov in a more transparent way. This is the case of Nadathur 's paper (2000) that obtained anew the conservativity for the first four of Orevkov's classes, without equality we must point out, and as a side-product of a study with a different purpose. The main purpose of Nadathur's work was not, in fact, a systematic study of the constructive content of classical theories, but an investigation of conditions that are sufficient for restricting sequents to single succedent form, to obtain goal-directedness in proof search, and the associated proposal of what he called uniform proofs as a foundation for logic programming.

In a similar direction of investigation, Schwichtenberg and Senjak (2013) proved that if $A$ is a formula without implications and $\Gamma$ consists of formulas that contain disjunctions and falsity only negatively and implications only positively, then classical derivability yields minimal derivability. Instead of sequent calculus, their approach uses natural deduction that is privileged because of its computational meaning through the Curry-Howard isomorphism, and a method for eliminating all the occurences of stability axioms, i.e., elimination of double negation, in the fragment considered.

A G3-style sequent calculus, as in Nadathur's work, is used by Ishihara (2000) to obtain a conservativity result of classical over intuitionistic theories. The result is obtained by defining inductively four classes $Q, R, J$, and $K$ of formulas and proving that if a formula in $K$ is classically provable from a collection of formulas of $Q$, then it is intuitionistically provable. Ishihara (2013) extended his previous results by introducing $\exists$ and $\forall$ in the inductive clauses for the classes $R$ and $J$, respectively. Both papers use a generalization of the double negation translation to obtain the stated results.

A famous conservative class is the one given by geometric implications, with the conservativity result known as the first-order Barr theorem. The result states that if a geometric implication is provable classically in a geometric theory, then it can be proved intuitionistically, or even minimally if there are no negative occurrences of falsity. Negri (2003) proved that the result reduces to a proof-theoretic triviality when a G3-style sequent calculus is used and geometric implications translated into extra rules the addition of which maintains 
the structural properties of the calculus: a classical derivation of a geometric implication in such a system is already an intuitionistic or minimal one. The result has been then generalized in Negri (2014) for theories axiomatized by formulas that do not contain implications or universal quantifiers in their negative part. Here, the proof mechanism still exploited the structural properties of G3-calculi and the translation of axioms into sequent rules, but with systems of rules rather than plain rules to account for an arbitrary nesting of quantifiers.

In this paper, we show how the appropriate choice of a sequent calculus, G3c, G3i, and G3m with the treatment of equality as introduced in Negri and von Plato (2001), greatly simplifies the conservativity proofs: equality is a part of the calculus and all the structural properties are maintained by the addition. The results are given in their original formulations, with the classes defined in terms of absence of certain logical constants in the positive or negative parts of sequents. For five of the seven classes the results are strengthened to height-preserving statements; for them, we do not give proof transformations but actually show that, with the appropriate calculus, the classical proofs are themselves intuitionistic or minimal proofs. The classical and the intuitionistic proofs have the same tree structure and the same rules employed, the only difference being the addition of the principal formula in the premisses of the left implication rule.

Some remarks on the converse result established by Orevkov and the extension to more general theories than the theory of equality are presented in the conclusion.

\section{Preliminaries}

We refer to Troelstra and Schwichtenberg (2000) and Negri and von Plato (2001, 2011) for the necessary background on sequent calculus. We shall in particular use the multi-succedent sequent calculi G3c for classical logic, G3i for intuitionistic logic, and G3m for minimal logic, defined as below (the two latter calculi are called m-G3i and m-G3m in Troelstra and Schwichtenberg (2000, p. 83), whereas Negri and von Plato (2001) follows Dragalin's formulation of the multi-succedent intuitionistic calculus, with a single-succedent left premiss of $L \supset$.

In the initial sequents, $P$ is an arbitrary atomic formula. Greek upper case letters $\Gamma, \Delta$ stand for arbitrary multisets of formulas. The restriction in $R \forall$ is that $y$ must not occur free in $\Gamma, \forall x A$ (and $\Delta$, for G3c). The restriction in $L \exists$ is that $y$ must not occur free in $\exists x A, \Gamma, \Delta$. We may summarize these conditions by the requirement that $y$ must not occur free in the conclusion of the two rules.

The calculus G3m for minimal logic is obtained from G3i by replacing $L \perp$ with the initial sequents

$$
\perp, \Gamma \Rightarrow \Delta, \perp
$$




\section{Initial sequents:}

$P, \Gamma \Rightarrow \Delta, P$

\section{Logical rules:}

$\frac{A, B, \Gamma \Rightarrow \Delta}{A \& B, \Gamma \Rightarrow \Delta} L \&$

$\frac{A, \Gamma \Rightarrow \Delta \quad B, \Gamma \Rightarrow \Delta}{A \vee B, \Gamma \Rightarrow \Delta} L \vee$

$\frac{\Gamma \Rightarrow \Delta, A \quad B, \Gamma \Rightarrow \Delta}{A \supset B, \Gamma \Rightarrow \Delta} L \supset$

$\overline{\perp, \Gamma \Rightarrow \Delta}{ }^{L \perp}$

$\frac{A(t / x), \forall x A, \Gamma \Rightarrow \Delta}{\forall x A, \Gamma \Rightarrow \Delta} L \forall$

$\frac{A(y / x), \Gamma \Rightarrow \Delta}{\exists x A, \Gamma \Rightarrow \Delta} L \exists$

$\frac{A \supset B, \Gamma \Rightarrow \Delta, A \quad B, \Gamma \Rightarrow \Delta}{A \supset B, \Gamma \Rightarrow \Delta} L$

\section{G3c}

$\frac{\Gamma \Rightarrow \Delta, A \quad \Gamma \Rightarrow \Delta, B}{\Gamma \Rightarrow \Delta, A \& B} R \&$

$$
\frac{\Gamma \Rightarrow \Delta, A, B}{\Gamma \Rightarrow \Delta, A \vee B} R \vee
$$

$\frac{A, \Gamma \Rightarrow \Delta, B}{\Gamma \Rightarrow \Delta, A \supset B} R$

$\frac{\Gamma \Rightarrow \Delta, A(y / x)}{\Gamma \Rightarrow \Delta, \forall x A} R \forall$

$\frac{\Gamma \Rightarrow \Delta, \exists x A, A(t / x)}{\Gamma \Rightarrow \Delta, \exists x A} R \exists$

\section{G3i}

$$
\begin{aligned}
& \frac{A, \Gamma \Rightarrow B}{\Gamma \Rightarrow \Delta, A \supset B} R \supset \\
& \frac{\Gamma \Rightarrow A(y / x)}{\Gamma \Rightarrow \Delta, \forall x A} R \forall
\end{aligned}
$$


All the structural rules (weakening, contraction and cut) are admissible in G3c, in G3i, and in G3m. The calculi are thus complete for classical, intuitionistic, and minimal first-order logic, respectively.

Orevkov's conservativity results are proved for predicate calculi with equality, so we need to extend the above G3 calculi with equality. This should be done in a way suitable to maintaining the structural properties of the purely logical calculi and for this purpose we follow the method of Negri and von Plato (2011, Section 6.3) and extend it to treat substitution of equals not just in predicates but also in functions.

We recall that axiomatic presentations of predicate logic with equality assume a primitive relation $a=b$ with the axiom of reflexivity, $a=a$, and the replacement scheme, $a=b \& A(a / x) \supset A(b / x)$, from which transitivity and symmetry are derivable (through Euclidean transitivity). In the standard treatment in sequent calculus (as in Troelstra and Schwichtenberg 2000, p. 128), one allows derivations to start with sequents of the following form where $P$ is an atomic formula:

$$
\Rightarrow a=a \quad a=b, P(a) \Rightarrow P(b)
$$

By Gentzen's "extended Hauptsatz," cuts can be reduced to cuts on such sequents, but full cut elimination fails. For example, there is no cut-free derivation of symmetry. Weakening and contraction must also be assumed.

An alternative way of adding equality and the replacement scheme consists in the method of axioms in the context. This is the method followed by Orevkov. By this method, the structural properties are maintained, but the presence of the universal closure of the axioms in the context introduces an element of perturbation to proof analysis. For example, conservativity of predicate logic with equality over predicate logic (cf. Negri and von Plato 2011, Section 6.3) could not be proved by this way of presenting the theory of equality.

To deal with equality, we first restrict the replacement scheme to atomic predicates $P, Q, R, \ldots$ and then convert the reflexivity and replacement axioms into the following rules

$$
\frac{a=a, \Gamma \Rightarrow \Delta}{\Gamma \Rightarrow \Delta} \operatorname{Ref} \quad \frac{a=b, P(a), P(b), \Gamma \Rightarrow \Delta}{a=b, P(a), \Gamma \Rightarrow \Delta} \operatorname{Repl}
$$

Substitution of equals in $n$-ary functions is a rule of simultaneus substitution, ${ }^{1}$ that corresponds to the axiom

$$
a_{1}=b_{1} \& \ldots \& a_{n}=b_{n} \supset f\left(a_{1}, \ldots, a_{n}\right)=f\left(b_{1}, \ldots, b_{n}\right)
$$

\footnotetext{
${ }^{1}$ For a concrete theory with equality where such a rule, formulated in a natural deduction style, is used, see the treatment of the word problem for groupoids in Section 4.3 of Negri and von Plato (2011). That example also shows why the rule has to be given as a rule of simultaneous substitution on all arguments of the function.
} 
It takes the form

$$
\frac{f\left(a_{1}, \ldots, a_{n}\right)=f\left(b_{1}, \ldots, b_{n}\right), a_{1}=b_{1}, \ldots, a_{n}=b_{n}, \Gamma \Rightarrow \Delta}{a_{1}=b_{1}, \ldots, a_{n}=b_{n}, \Gamma \Rightarrow \Delta} \text { Sub }
$$

Rule $S u b$ is derivable from Ref and Repl, so it doesn't need to be taken as a primitive rule of the calculus for equality. To see this, consider a function of two arguments. We have the following derivation:

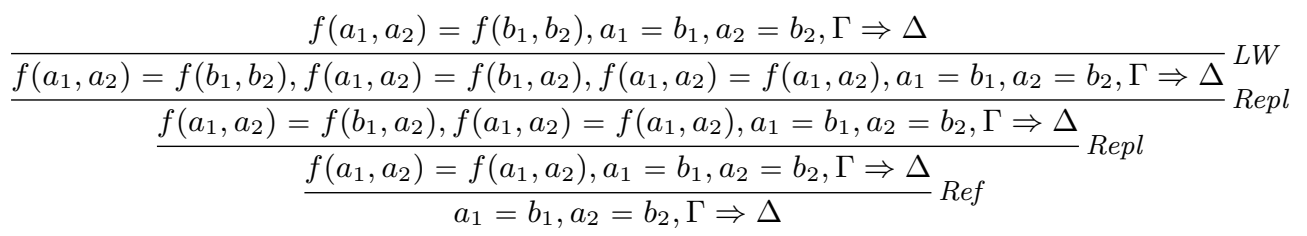

Here the lower and upper steps of Repl are obtained as instances of the general scheme with the predicates $P_{1}(x) \equiv f\left(a_{1}, a_{2}\right)=f\left(x, a_{2}\right)$ and $P_{2}(x) \equiv$ $f\left(a_{1}, a_{2}\right)=f\left(b_{1}, x\right)$, respectively. The proof is generalized by induction to functions of $n$ arguments.

When rules Ref and $R e p l$ are added to G3m, G3i, and G3c minimal, intuitionistic and classical predicate logic with equality are obtained, respectively. We shall denote such extensions by $\mathbf{G} \mathbf{3} \mathbf{m}^{=}, \mathbf{G} 3 \mathbf{i}^{=}$, and $\mathbf{G} \mathbf{3} \mathbf{c}^{=}$. We have:

Theorem 1 The following properties hold in $\mathbf{G} 3 \mathbf{m}^{=}, \mathbf{G} 3 \mathbf{i}^{=}$, and $\mathbf{G} 3 \mathbf{c}^{=}$:

$i$. The rules of weakening and contraction are height-preserving admissible, and cut is admissible.

ii. The replacement axiom $a=b, A(a / x) \Rightarrow A(b / x)$ is derivable for arbitrary A.

iii. The replacement rule

$$
\frac{a=b, A(a / x), A(b / x), \Gamma \Rightarrow \Delta}{a=b, A(a / x), \Gamma \Rightarrow \Delta} \operatorname{Repl}
$$

is admissible for an arbitrary predicate $A$.

Proof. See Negri and von Plato (2011, 6.14-6.16). QED.

Next, we obtain the conservativity of first-order logic with equality over firstorder logic also in the presence of functions. ${ }^{2}$

\footnotetext{
${ }^{2}$ The result (without functions) was already established in Negri and von Plato (2011). The proof given there, based on a prior elimination of the rule of reflexivity from all derivations of equality-free sequents, can be easily extended in the presence of functions, even with an explicit rule of substitution of equals. We shall however give here a shorter proof suggested by a referee.
} 
We shall use the standard notation (as in Negri and von Plato 2001)

$$
\mathbf{S} \vdash_{n} \Gamma \Rightarrow \Delta
$$

to indicate that the sequent $\Gamma \Rightarrow \Delta$ is derivable with a height of derivation at most $n$ in the sequent calculus $\mathbf{S}$.

Lemma 2 If $\mathbf{G 3} \mathbf{c}^{=} \vdash_{n} a_{1}=a_{1}, \ldots, a_{m}=a_{m}, \Gamma \Rightarrow \Delta$, with $\Gamma, \Delta$ containing no equalities and $m \geq 0$, then $\mathbf{G 3 \mathbf { c }} \vdash_{n} \Gamma \Rightarrow \Delta$.

Proof. By induction on $n$. If $n=0, a_{1}=a_{1}, \ldots, a_{m}=a_{m}, \Gamma \Rightarrow \Delta$ is either an initial sequent or conclusion of $L \perp$. In the former case, by the assumption on $\Delta$, none of the equalities is principal, and therefore also $\Gamma \Rightarrow \Delta$ is initial. In the latter, $\perp$ is in $\Gamma$ and the conclusion follows.

If $n>0$ we consider the last rule applied, which gives one of the three cases: 1. Logical rule; 2. Ref; 3. Repl.

In the first case, we apply the induction hypothesis to the premiss(es) of the rule, and then the rule.

In the second, if $a=a$ is the atomic formula in the premiss of Ref, we apply the induction hypothesis to the sequent $a=a, a_{1}=a_{1}, \ldots, a_{m}=a_{m}, \Gamma \Rightarrow \Delta$.

In the third case we have a derivation that ends with

$$
\frac{a_{1}=a_{1}, \ldots, a_{k}=a_{k}, a=b, P(b), P(a), \Gamma^{\prime} \Rightarrow \Delta}{a_{1}=a_{1}, \ldots, a_{k}=a_{k}, a=b, P(a), \Gamma^{\prime} \Rightarrow \Delta} \text { Repl }
$$

If $P(a)$ is an equality predicate, it can be only of the form $a=a$ by the assumption on the given sequent. Similarly we must have $a \equiv b$, and therefore also the premiss consists of reflexivity atoms and an equality-free context. In this case we proceed as in the previous one. Else, since $a \equiv b$ by assumption, also $P(a) \equiv P(b)$. By height-preserving admissibility of contraction we obtain G3c ${ }^{=} \vdash_{n-1} \quad a_{1}=a_{1}, \ldots, a_{m}=a_{m}, a=b, P(a), \Gamma \Rightarrow \Delta$ and by induction hypothesis $\mathbf{G 3 \mathbf { c }} \vdash_{n-1} P(a), \Gamma \Rightarrow \Delta$. QED

As a special case in which the number of reflexivity atoms is zero, we obtain the conservativity theorem of predicate logic with equality over predicate logic:

Theorem 3 If $\Gamma \Rightarrow \Delta$ contains no equalities and is derivable in $\mathbf{G 3} \mathbf{c}^{=}$, then it is derivable in $\mathbf{G 3 c}$.

\section{Orevkov's theorems on Glivenko sequent classes revisited}

We follow Orevkov's notation and denote by $\circ^{+}$positive and by $\circ^{-}$negative occurrences of the connective or quantifier $\circ$ in a sequent.

In re-establishing Orevkov's results we shall also pay attention to the height of derivations to show that stronger results are achieved when G3 calculi are used. 
Theorem 4: Conservativity for class 1. Suppose that $\Gamma \Rightarrow \Delta$ does not contain $\supset^{+}$or $\forall^{+}$and that $\mathbf{G 3} \mathbf{c}^{=} \vdash_{n} \Gamma \Rightarrow \Delta$. Then $\mathbf{G} 3 \mathbf{i}^{=} \vdash_{n} \Gamma \Rightarrow \Delta$. If in addition $\Gamma \Rightarrow \Delta$ does not contain $\perp^{-}$, then $\mathbf{G} 3 \mathbf{m}^{=} \vdash_{n} \Gamma \Rightarrow \Delta$.

Proof. By the assumption, the given derivation contains no application of $R \supset$ or of $R \forall$, therefore it gives directly a derivation in $\mathbf{G} 3 \mathbf{i}^{=}$by the heightpreserving addition of the principal formula in the left premiss whenever $L \supset$ is applied.

If $\Gamma \Rightarrow \Delta$ does not contain $\perp^{-}$, then $L \perp$ is never used and the intuitionistic derivation is indeed a minimal one. QED

Theorem 5: Conservativity for class 2. Suppose that $\Gamma \Rightarrow A$ does not contain $\supset^{+}$or $\vee^{-}$and that $\mathbf{G} 3 \mathbf{c}^{=} \vdash_{n} \Gamma \Rightarrow A$. Then $\mathbf{G} 3 \mathbf{i}^{=} \vdash_{n} \Gamma \Rightarrow A$.

The result is an immediate consequence of the following lemma: ${ }^{3}$

Lemma 6 Suppose that $\mathbf{G 3 c}=\vdash_{n} \Gamma \Rightarrow \Delta$ where $\Gamma \Rightarrow \Delta$ does not contain $\supset^{+}$ or $\vee^{-}$. Then $\begin{cases}\text {there is } A \text { in } \Delta \text { such that } \mathbf{G} 3 \mathbf{i}^{=} \vdash_{n} \Gamma \Rightarrow A & \text { if } \Delta \neq \emptyset \\ \mathbf{G 3}^{=}{ }^{=} \vdash_{n} \Gamma \Rightarrow \Delta & \text { if } \Delta=\emptyset\end{cases}$

Proof. By induction on the height of the derivation.

If $\Gamma \Rightarrow \Delta$ is an initial sequent, then the principal formula will do; if it is conclusion of $L \perp$, then any formula from $\Delta$ or the empty set gives the desired conclusion.

If the last rule is $L \&$, with premiss $C, D, \Gamma^{\prime} \Rightarrow \Delta$ and $\Delta \neq \emptyset$, by induction hypothesis we find $A$ in $\Delta$ such that $C, D, \Gamma^{\prime} \Rightarrow A$ is derivable in $\mathbf{G} 3 \mathbf{i}^{=}$(with height diminished by one) and by $L \&$ we have derivability of $C \& D, \Gamma^{\prime} \Rightarrow A$ (with the same height as the original derivation). The case with $\Delta=\emptyset$ is proved similarly.

If the last rule is $R \&$, with premisses $\Gamma \Rightarrow A_{1}, \Delta^{\prime}$ and $\Gamma \Rightarrow A_{2}, \Delta^{\prime}$, there are four cases to consider, according to the provenance of the formulas $B_{1}$ and $B_{2}$ given by the inductive hypothesis. If one is $A_{1}$ and the other $A_{2}$, we conclude by applying $R \&$. If one or both are in $\Delta^{\prime}$ we just take that derivation to conclude.

If the last rule is $R \vee$, with premiss $\Gamma \Rightarrow \Delta^{\prime}, A_{1}, A_{2}$, by inductive hypothesis there is $A$ in $\Delta^{\prime}, A_{1}, A_{2}$ such that $\mathbf{G} 3 \mathbf{i}^{=} \vdash_{n-1} \Gamma \Rightarrow A$. If $A$ is in $\Delta^{\prime}$ we have already the conclusion. If $A$ is either $A_{1}$ or $A_{2}$, then by height-preserving admissibility of weakening we obtain $\mathbf{G} 3 \mathbf{i}^{=} \vdash_{n-1} \Gamma \Rightarrow A_{1}, A_{2}$ and the conclusion G3i ${ }^{=} \vdash_{n} \Gamma \Rightarrow A_{1} \vee A_{2}$ follows by a step of $R \vee$. (Observe that the argument would break apart if rule $L \vee$ were allowed, because the two formulas given by the inductive hypothesis in the case $\Delta \neq \emptyset$ could be different, thus preventing from restoring the original antecedent by application of $L \vee$.)

\footnotetext{
${ }^{3}$ It was observed by a referee that the proof below gives a stronger statement than just a "classical disjunction property" as in the original submission, with the benefit of an immediate proof of Theorem 5 .
} 
If the last rule is $L \supset$, with premisses $\Gamma^{\prime} \Rightarrow C, \Delta$ and $D, \Gamma^{\prime} \Rightarrow \Delta$, and $\Delta \neq \emptyset$, by inductive hypothesis we find $A_{1}$ in $C, \Delta$ and $A_{2}$ in $\Delta$ such that $\Gamma^{\prime} \Rightarrow A_{1}$ and $D, \Gamma^{\prime} \Rightarrow A_{2}$. We distinguish the two cases for $A_{1}$. In the first case, $A_{1} \equiv C$ and we have the following derivation, where we use the height-preserving admissible rules of left and right weakening:

$$
\frac{\frac{\Gamma^{\prime} \Rightarrow C}{C \supset D, \Gamma^{\prime} \Rightarrow C, A_{2}}{ }^{L W, R W} \quad D, \Gamma^{\prime} \Rightarrow A_{2}}{C \supset D, \Gamma^{\prime} \Rightarrow A_{2}} L
$$

In the second case, from $\Gamma^{\prime} \Rightarrow A_{1}$ we obtain the conclusion $C \supset D, \Gamma^{\prime} \Rightarrow A_{1}$ by height-preserving admissibility of left weakening. If $\Delta=\emptyset$, we have a derivation ending with

$$
\frac{\Gamma^{\prime} \Rightarrow C \quad D, \Gamma^{\prime} \Rightarrow}{C \supset D, \Gamma^{\prime} \Rightarrow}
$$

By induction hypothesis on the two premisses we obtain the following derivation in $\mathbf{G} 3 \mathbf{i}^{=}$

$$
\frac{\frac{\Gamma^{\prime} \Rightarrow C}{C \supset D, \Gamma^{\prime} \Rightarrow C}}{C \supset D, \Gamma^{\prime} \Rightarrow} \quad D, \Gamma^{\prime} \Rightarrow L \supset
$$

with the same derivation height. (Observe that the addition of $R \supset$ among the rules allowed in the derivation would break down the induction if the formula given by the induction hypothesis in the case $\Delta \neq \emptyset$ is in the context of the premiss.)

If the last rule is a rule for equality, we simply apply the inductive hypothesis and then the rule.

The cases in which the last rule is a rule for the quantifiers are straightforward: first the inductive hypothesis is applied and then, possibly, in case the selected formula is the active formula of the rule, the rule is applied again (after an additional height-preserving step of $R W$ in the case of $R \exists$ ) to restore the conclusion. The variable conditions, if any, are satisfied because the application is performed with a context which is included in the original one. QED

Corollary 7 Suppose that $\Gamma \Rightarrow \Delta$ does not contain $\supset^{+}$or $\bigvee^{-}$and that $\mathbf{G} 3 \mathbf{c}^{=} \vdash_{n}$ $\Gamma \Rightarrow \Delta$. Then $\mathbf{G} 3 \mathbf{i}^{=} \vdash_{n} \Gamma \Rightarrow \Delta$. If in addition $\Gamma \Rightarrow \Delta$ does not contain $\perp^{-}$, then $\mathbf{G} 3 \mathbf{m}^{=} \vdash_{n} \Gamma \Rightarrow \Delta$.

Proof. The first part is immediate consequence of Lemma 6. The proof that the additional assumption on $\perp$ gives a minimal derivation is as for class 1 . QED

As a special case of the conservativity established in the corollary, we obtain the sequent calculus version of the result proved by Schwichtenberg and Senjak (2013): 
Corollary 8 If $A$ is a formula without implications and $\Gamma$ consists of formulas containing disjunction and falsity only negatively, and implications only positively, then classical derivability implies minimal derivability.

Theorem 9: Conservativity for class 3. Suppose that $\Gamma \Rightarrow A$ does not contain $\supset^{+}$or $\forall^{-}$and that $\mathbf{G} 3 \mathbf{c}^{=} \vdash \Gamma \Rightarrow A$. Then $\mathbf{G} 3 \mathbf{i}^{=} \vdash \Gamma \Rightarrow A$. If in addition $\Gamma \Rightarrow A$ does not contain $\perp^{-}$, then $\mathbf{G} 3 \mathbf{m}^{=} \vdash \Gamma \Rightarrow A$.

By the following lemma, the proof of conservativity for class 3 can be reduced to that for the second class. Here the proof follows Orevkov's method of permutation of rules. The idea is to divide all the rules that can possibly be involved in a derivation of a sequent in the class in two groups. The first group $G_{1}$ consists of the equality rules and the right rules that can be used in the derivation of the sequent, that is, $G_{1} \equiv\{R e f, R e p l, R \&, R \vee, R \exists, R \forall\}$. The second group $G_{2}$ consists of all the left rules that can possibly be used in the derivation, i.e. $G_{2} \equiv\{L \&, L \vee, L \supset, L \exists\}$. It is then proved that derivations of sequents in the third class can be taken to a form in which any rules from the second group follows any rule from the first group. The conclusion will follow because for sequents in the first part of the derivation we have conservativity by the result for class 2. The second part consists instead of rules that are identical (modulo use of height-preserving weakening for $L \supset$ ), in the classical, intuitionistic, and minimal calculus.

Lemma 10 Suppose that $\Gamma \Rightarrow \Delta$ does not contain $\supset^{+}$or $\forall^{-}$and that $\mathbf{G 3 c}=\vdash$ $\Gamma \Rightarrow \Delta$. Then there is a derivation of $\Gamma \Rightarrow \Delta$ with the property that all the rules in group $G_{1}$ precede all the rules in the group $G_{2}$.

Proof. In general, permutation of rules is straighforward in case the principal formula of the upper rule is not an active formula of the lower rule, and this applies to each of the desired permutations: for the permutations below the equality rules, observe that these rules have atomic formulas as active formulas; for the permutations below the other rules, note that these rules do not include $R \supset$ and thus their active formulas are on the right-hand side of sequents. Observe that in the permutation for rules below $R \forall$ the variable condition is satisfied: the only case to be analyzed with a potential clash of variable is the one in which $L \exists$ is permuted below $R \forall$. In this case, the eigenvariable of $L \exists$ is necessarily distinct from that of $R \forall$ so the variable conditions are satisfied when the two rules are permuted. QED

Theorem 11 : Conservativity for class 3-multi. Suppose that $\Gamma \Rightarrow \Delta$ does not contain $\supset^{+}$or $\forall^{-}$and that $\mathbf{G} \mathbf{3} \mathbf{c}^{=} \vdash \Gamma \Rightarrow \Delta$. Then $\mathbf{G} 3 \mathbf{i}^{=} \vdash \Gamma \Rightarrow \Delta$. If in addition $\Gamma \Rightarrow \Delta$ does not contain $\perp^{-}$, then $\mathbf{G 3}=\vdash \Gamma \Rightarrow \Delta$. 
Proof. By Corollary 7, any sequent in the part of the derivation tree with rules in $G_{1}$ is also derivable in $\mathbf{G} 3 \mathbf{i}^{=}$. The part with rules in $G_{2}$ maintains the derivation in $\mathbf{G} 3 \mathbf{i}^{=}$. The conclusion for $\mathbf{G} \mathbf{3} \mathbf{m}=$ is obtained as before. QED Next we have:

Theorem 12: Conservativity for class 4. Suppose that $\Gamma \Rightarrow A$ does not contain $\supset^{-}, \vee^{+}$, or $\exists^{+}$and that $\mathbf{G 3} \mathbf{c}^{=} \vdash_{n} \Gamma \Rightarrow A$. Then $\mathbf{G} 3 \mathbf{i}=\vdash_{n} \Gamma \Rightarrow A$. If in addition $\Gamma \Rightarrow A$ does not contain $\perp^{-}$, then $\mathbf{G} \mathbf{3 m}=\vdash_{n} \Gamma \Rightarrow A$.

Proof. Observe that the only rules of $\mathbf{G 3} \mathbf{c}^{=}$that can produce a singlesuccedent conclusion from a multi-succedent premiss are $R \vee, L \supset$, and $R \exists$. It therefore follows from the assumed restrictions that all the sequents in the derivation of $\Gamma \Rightarrow A$ are necessarily single-succedent ones, thus all the instances of $R \supset$ and of $R \forall$ in the derivation are actually correct instances of these rules in $\mathbf{G 3 i} \mathbf{i}$, hence in $\mathbf{G} 3 \mathbf{i}^{=}$. The result then follows by a straightforward induction on the height of the derivation. QED

The following lemma and its corollary have restrictions that permit to relax the restrictions on $R \supset$ of the classes 1,2, 3 to restrictions which ban only the applications of $R \supset$ in which the succedent is not $\perp$. More specifically, the classes are defined as the classes 1,2, 3 with the restriction on positive implications replaced by a restriction on positive implications with succedent which is not $\perp$ and all negative implications (the genuine ones and negations). The statement of the lemma (but not the idea behind the proof) differs from that in Orevkov where a calculus with primitive negation is used; the calculi that we use have instead a defined negation, and therefore the restrictions are here formulated in terms of negative or positive occurrences of $\perp$, rather than on the polarity of occurrences of negation. ${ }^{4}$ The requirement that a sequent $\Gamma \Rightarrow \Delta$ does not contain negations in negative parts is clearly not equivalent to the one that it does not contain $\perp^{-}$. However, we are interested in proving a conservativity result, so we can further assume without loss of generality that $\Gamma$ does not contain $\perp$ because, if it did, the result would trivially hold.

Lemma 13 Suppose that $\Gamma \Rightarrow \Delta$ does not contain $\perp^{-}, \vee^{+}$, or $\supset^{+}$and that $\Delta$ is empty or $\perp$ occurs positively in all the formulas of $\Delta$. Then $\Gamma \Rightarrow \Delta$ is not derivable in $\mathbf{G} \mathbf{3} \mathbf{c}^{=}$.

Proof. Clearly, $\Gamma \Rightarrow \Delta$ is not an initial sequent (atomic formulas do not contain $\perp$ ), nor an instance of $L \perp$. We consider all the possible ways of decomposing

\footnotetext{
${ }^{4}$ The statement of Lemma 4.3 in the English translation of Orevkov's article contains a misprint: the sentence "the succedent of $S$ is empty or occurs positively in all of the subformulas of the succedent of $S$ " should be "the succedent of $S$ is empty or negation occurs positively in all of the formulas of the succedent of $S$." Our guess was confirmed by a comparison with the Russian original, which is correctly translated into: "Lemma 4.3. Whatever be the sequence $\mathrm{S}$ of the calculus $\mathrm{C}^{*}$, if there are no occurrences of logical connectives of type $\neg^{-}, \mathrm{V}^{+}$and $\supset^{+}$ and if the succedent of $\mathrm{S}$ is empty or $\neg$ occurs positively in all formulas of the succedent of $\mathrm{S}$, then the sequent $\mathrm{S}$ is not derivable in the calculus $\mathrm{C}^{*} . "$
} 
the sequent. The possible rules are Ref, Repl, $L \&, R \&, L \vee, L \supset, L \exists, R \exists, L \forall$, $R \forall$ and it is seen by inspection on each of these that if the conclusion of the rule satisfies the assumptions, also the premiss, or at least one of the two premisses, does. Well foundedness of the decomposition procedure and invertibility of the rules gives the conclusion. QED

Corollary 14 Suppose that $\Gamma \Rightarrow A$ does not contain $\neg^{-}, \vee^{+}, \supset^{+}$, or $\supset^{-}$, that $\Gamma$ does not contain $\perp$, but $\Gamma \Rightarrow A$ contains a positive occurrence of negation. Then $\Gamma \Rightarrow A$ is not derivable in $\mathbf{G} \mathbf{3} \mathbf{c}^{=}$.

Proof. Because of the restrictions on implication, negation, hence $\perp$, is found positively in $A$, so the sequent satisfies the conditions of the above lemma. QED

Corollary 15 If a sequent $\Gamma \Rightarrow A$ that does not contain $\neg^{-}, \vee^{+}, \supset^{+}$, or $\supset^{-}$ but contains a positive occurrence of negation, is derivable in $\mathbf{G} 3 \mathbf{c}^{=}$then it is also derivable in $\mathbf{G} 3 \mathbf{i}^{=}$.

Proof. If $\Gamma$ contains $\perp$, the result is obvious because the sequent is a conclusion of $L \perp$. If $\Gamma$ does not contain $\perp$, the result holds vacuously as a consequence of Corollary 14. QED

Conservativity for Orevkov' classes 5,6 , and 7 is then trivially obtained. We state (and prove) these results in detail:

Theorem 16: Conservativity for class 5. Suppose that $\Gamma \Rightarrow A$ does not contain $\supset^{-}, \mathrm{V}^{+}, \forall^{+}$and does not contain $\supset^{+}$with the possible exception of negations and that $\mathbf{G} 3 \mathbf{c}^{=} \vdash_{n} \Gamma \Rightarrow A$. Then $\mathbf{G} 3 \mathbf{i}^{=} \vdash_{n} \Gamma \Rightarrow A$.

Proof. If $\Gamma \Rightarrow A$ does not contain positive negations, the class is a subclass of class 1 , and the result follows. If it contains positive negations, it holds vacuosly by Corollary 15. If $\Gamma$ contains $\perp$, the result is obvious because the sequent is a conclusion of $L \perp$. If $\Gamma$ does not contain $\perp$, the result holds by Corollary 15 . QED

Theorem 17: Conservativity for class 6. Suppose that $\Gamma \Rightarrow A$ does not contain $\supset^{-}, \mathrm{V}^{+}, \mathrm{V}^{-}$and does not contain $\supset^{+}$with the possible exception of negations and that $\mathbf{G 3} \mathbf{c}^{=} \vdash_{n} \Gamma \Rightarrow A$. Then $\mathbf{G} 3 \mathbf{i}^{=} \vdash_{n} \Gamma \Rightarrow A$.

Proof. As above, with class 2 in place of class 1. QED

Theorem 18: Conservativity for class 7. Suppose that $\Gamma \Rightarrow A$ does not contain $\supset^{-}, \mathrm{V}^{+}, \forall^{-}$and does not contain $\supset^{+}$with the possible exception of negations and that $\mathbf{G} 3 \mathbf{c}^{=} \vdash \Gamma \Rightarrow A$. Then $\mathbf{G} 3 \mathbf{i}^{=} \vdash \Gamma \Rightarrow A$.

Proof. As above, with class 3 in place of class 1. QED 


\section{Conclusion}

We have proved conservativity for classical over intuitionistic (or minimal) firstorder logic extended with the theory of equality for seven sequent classes. The direct methods of proof analysis in G3 sequent calculi give for all the classes transparent proofs and yield for five of them stronger, height-preserving, statements. The conversion of axioms into rules for the theory of equality also shows to what extent the specific form of the axioms of equality plays a role in the proofs. It is natural then to ask whether it is possible to extend the result to more general theories. As observed by a referee, all the results in Section 3 continue to hold if we consider any Horn theory in place of the theory of equality. The reason is that any such theory can be converted into rules that follow the regular rule scheme and have at most one premiss: in all the proofs we require only that the added rules are the same for the classical, intuitionistic, and minimal calculus, and have only atomic formulas as active and principal; further, for the validity of Lemma 6, we need to exclude positive disjunctions in the theory, or equivalently, rules with several premisses. We further observe that we can extend the results to geometric theories with no disjunctions, that is, to extensions by rules of the form ${ }^{5}$

$$
\frac{\bar{Q}(\bar{y} / \bar{x}), \bar{P}, \Gamma \Rightarrow \Delta}{\bar{P}, \Gamma \Rightarrow \Delta} H G R S
$$

where $\bar{Q}$ and $\bar{P}$ indicate the multisets of atomic formulas $Q_{1}, \ldots Q_{n}$ and $P_{1}$, $\ldots, P_{m}$, respectively, and the eigenvariables $\bar{y}$ of the premiss are not free in the conclusion.

All the proofs are identical to those for the theory of equality, but there is a potential clash of variables in the permutation of the rules for quantifiers past the geometric rules in the proof of Lemma 10. The only case of permutability to be checked is thus the one of $L \exists$ past HGRS. This is unproblematic because of the variable condition of $L \exists$. No critical cases arise because we do not need to permute $R \exists$ (the rule is in $G_{1}$ ) nor $L \forall$ (ruled out by the assumption on the endsequent).

Orevkov's results also concern with a converse to the conservativity of the seven classes, that can be stated as follows: if for a class of sequents classical derivability yields intuitionistic derivability, then the class is contained in one of the seven classes. Orevkov proves the result by contradiction: from the assumptions that a class is not contained in any of the seven classes, a list of possible violations to the defining properties of each class is obtained. For example, it is not in class 1 if it either contains positive occurrences of implications or of

\footnotetext{
${ }^{5}$ The proof-theoretic properties of extensions of sequent calculi by rules of such form are investigated in Negri (2003). A systematic treatment of sequent calculus extended by geometric rules is also found in Negri and von Plato (2011), Chapter 8, with examples of geometric theories without disjunctions detailed in Chapter 5.
} 
universal quantifiers. An enumeration of all the possible cases (obtained from a conjunction of disjunctions of possibilities) gives different possibilities of coexisting connectives or quantifiers in the positive or negative part. The list is reduced by taking into account inclusions between some of the cases and the empty conservativity result for the last three classes. In the end, the result is reduced to showing that there are sequents corresponding to each remaining combination that are derivable classically but not intuitionistically. For this purpose, Orevkov first shows that derivations are reducible to appropriate forms (for which the denomination weeded and weakly weeded is introduced in the English translation of the article). These are derivations that do not contain excess applications of logical rules or of formulas and that obtain a characterization in terms of the order of application of rules. All such preparatory work is avoided with the use of the G3i calculus for intuitionistic logic, and all underivabilities are shown in a straightforward way by failed root-first proof search as in Section 4.3(b) of Negri and von Plato (2001).

Acknowledgements: I wish to thank Helmut Schwichtenberg for having brought to my attention Orevkov's conservativity results, Hajime Ishihara for a useful discussion on his approach to conservativity classes and Per Martin-Löf for the translation from Russian of a passage in the original version of Orevkov (1968). Two referees are gratefully acknowledged for their constructive and insightful suggestions.

\section{References}

Ishihara, H. (2000) A note on the Gödel-Gentzen translation, Mathematical Logic Quarterly, vol. 46, pp. 135-137.

Ishihara, H. (2013) Some conservative extension results on classical and intuitionistic sequent calculi. In Berger, U. et al. (eds) Logic, Construction, Computation, pp. 289-304, Ontos Verlag, Heusenstamm.

Nadathur, G. (2000) Correspondence between classical, intuitionistic and uniform provability. Theoretical Computer Science, vol. 232, pp. 273-298.

Negri, S. (2003) Contraction-free sequent calculi for geometric theories, with an application to Barr's theorem. Archive for Mathematical Logic, vol. 42, pp. 389-401.

Negri, S. (2014) Proof analysis beyond geometric theories: from rule systems to systems of rules. Journal of Logic and Computation, doi: 10.1093/logcom/exu037.

Negri, S. and J. von Plato (2001) Structural Proof Theory. Cambridge University Press.

Negri, S. and J. von Plato (2011) Proof Analysis. Cambridge University Press.

Orevkov, V. P. (1968) On Glivenko sequent classes. Proc. Steklov Inst. Math., vol. 98, pp. 147-173. Translated from the Russian original V. P. Orevkov, Glivenko's sequence classes, Logical and logical-mathematical calculus, Part I. Trudy Mat. Inst. Steklov, vol. 98, 1968, pp. 131-154. 
Schwichtenberg, H. and C. Senjak (2013) Minimal from classical proofs. Annals of Pure and Applied Logic, vol. 164, pp. 740-748.

Troelstra, A. and H. Schwichtenberg (1996) Basic Proof Theory. Cambridge University Press, second edition 2000. 